anaesthetising each tooth, based on the best available evidence in the literature, including how to proceed when local anaesthetic failure occurs and the best supplementary techniques for each situation. New local anaesthetic agents and equipment are described, however, sufficient evidence does not yet exist to support their use.

In conclusion, a lot of research has gone into the writing of this book. It would be useful as a reference for any dentist having trouble with failed local anaesthesia in restorative and endodontic procedures and also for those with a special interest in local anaesthesia and pain management.

M. Devine

\section{TEN CATE'S ORAL HISTOLOGY,} 8TH EDITION

\begin{tabular}{|l|l}
\hline 8 & A. Nanci \\
\hline TenCate's Oral & UK: Elsevier \\
Histology & price £74.99; pp 400 \\
US & ISBN 9780323078467 \\
\hline
\end{tabular}

This textbook, aimed at undergraduate students, suits its purpose perfectly, as it is straightforward and easy to understand.

It covers oral histology over 15 chapters, with molecular concepts highlighted throughout to increase understanding in certain areas, such as when cell function is discussed. Chapters covered include the structure of the oral tissues, embryology of the head and neck and tooth development, as well as more general chapters on the structures common to various cells in the body and their functions, and tissue repair and regeneration. There is a new chapter on facial growth and development but this is brief and only provides an overview. The reader is subsequently directed to another text, which is cited, for more in-depth information.

The book is designed so that each chapter can be read discretely, with a brief introduction at the start and an outline similar to a table of contents. There is also the opportunity to register with the Evolve learning system online for access to a free study tool with review questions, exercises and an enhanced learning experience.
One of the best features of this book is the use of images and diagrams. There is a mixture of colour and monochrome images and they complement the text well. Not only do they aid understanding but they make the book more engaging. The diagrams and histological images help the reader to gauge principles that would be hard to picture with just text and imagination. Scanning electron micrographs offer excellent detail and are well annotated for an undergraduate understanding. The diagram on the development of the functional dentition from inception to the permanent dentition, although regarding content is excellent, was a little confusing and hard to decipher.

The book mentions current research on the bioengineering of teeth and advances in implantology with scope for further reading in the recommended reading section, which is present at the end of every chapter.

This book on the fundamental elements of dentistry is interesting, understandable and a comprehensive source of information for dental students.

N. Miller

\section{ORAL AND MAXILLOFACIAL PATHOLOGY: A RATIONALE FOR DIAGNOSIS AND TREATMENT, 2ND EDITION}

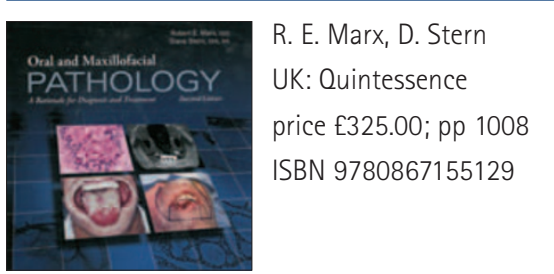

This updated second edition provides a comprehensive reference for oral and maxillofacial specialists and related professionals with over 500 new colour photographs, updates on treatments and management of over 400 conditions and diseases, including more recent internationally recognised diseases such as bisphosphonate related osteonecrosis, not incorporated in the first 2003 publication. The text is presented over two cased volumes making it a more manageable and practical reference source given the length of the text.

Eleven chapters over the two volumes cover a vast array of topics from the more commonly seen diseases and lesions such as candidosis to those less prevalent such as verrucous carcinoma. These are ordered in a surgical sieve like classification approach covering a range of topics from inflammatory diseases, infectious diseases, and immune-based diseases to fibro-osseous diseases, salivary glands and pigmented lesions. A particularly interesting chapter is solely dedicated to osteoradionecrosis, detailing its prevention, management, treatment and guidelines for dental treatment before, during and post radiotherapy to the head and neck in a general practice setting as well as hospital. The opening chapter provides a useful introduction to biopsy principles and techniques, with the final chapters providing a guide listing outdated and new terms to prevent confusion over terminology and an interesting brief biography of 57 notable people who have influenced and contributed to the profession.

Each of the 425 conditions are described under several headings including: clinical presentation, differential diagnosis, diagnostic work up, histopathology, treatment and prognosis. This arrangement of the text allows for easy location of relevant information and for the reader to assess specific information as detailed or briefly as required. The treatment aspects vary from basic tests such as appropriate radiographs relevant to general practice to more complex specialist investigations and treatment including step-bystep clinical photographs on surgical management where appropriate. As an American publication some of the guidelines differ, such as the guidance from the American Heart Association on prescribing prophylaxis for infective endocarditis for at risk patients.

This $30.7 \mathrm{~cm} \times 28.4 \mathrm{~cm} \times 8.1 \mathrm{~cm}$ hardback textbook containing 2,700 images including diagrams, histology slides, radiographs and photographs is a desirable reference guide to all things oral and maxillofacial. The text is especially appealing to maxillofacial surgeons and those on a training pathway alongside general dental practitioners wishing to build upon and develop their knowledge using a well written comprehensive text.

D. Lee 